\title{
Invasive Serotype 35B Pneumococci Including an Expanding Serotype Switch Lineage, United States, 2015-2016
}

\author{
Sopio Chochua, Benjamin J. Metcalf, Zhongya Li, Hollis Walker, \\ Theresa Tran, Lesley McGee, Bernard Beall
}

We used whole-genome sequencing to characterize 199 nonvaccine serotype $35 \mathrm{~B}$ pneumococcal strains that caused invasive pneumococcal disease (IPD) in the United States during 2015-2016 and related these findings to previous serotype 35B IPD data obtained by Active Bacterial Core surveillance. Penicillin-nonsusceptible 35B IPD increased during post-pneumococcal 7-valent conjugate vaccine years (2001-2009) and increased further after implementation of pneumococcal 13-valent conjugate vaccine in 2010 . This increase was caused primarily by the 35B/sequence type (ST) 558 lineage. 35B/ST558 and vaccine serotype 9V/ST156 lineages were implicated as cps35B donor and recipient, respectively, for a single capsular switch event that generated emergent 35B/ST156 progeny in 6 states during 2015-2016. Three additional capsular switch $35 \mathrm{~B}$ variants were identified, 2 of which also involved 35B/ST558 as cps35B donor. Spread of 35B/ ST156 is of concern in view of past global predominance of pathogenic ST156 vaccine serotype strains. Protection against serotype 35B should be considered in next-generation pneumococcal vaccines.

lthough the dramatic protective effect of the pneu-
mococcal 7-valent conjugate vaccine (PCV7) against
invasive pneumococcal disease (IPD) persisted a full de-
cade after its introduction in the United States in 2000, the
emergence of 19A and other non-PCV7 serotypes reduced
the overall benefit $(1,2)$. Before PCV7 implementation,
we observed only 2 different 35B lineages within Active
Bacterial Core surveillance (ABCs) (3), a population-
based, multistate program that assesses the effect of inva-
sive bacterial infections and is part of the Emerging Infec-
tions Program network of the Centers for Disease Control
and Prevention (CDC; Atlanta, GA, USA) (http://www.
cdc.gov/abcs/index.html). Both lineages were relatively

Author affiliation: Centers for Disease Control and Prevention,

Atlanta, Georgia, USA

DOI: http://dx.doi/10.3201/eid2306.170071 rare causes of IPD but were geographically widespread in the United States before and after PCV7 introduction (3). One 35B lineage was antimicrobial-susceptible and multilocus sequence type (MLST) 452 (35B/ST452), and the second strain was penicillin-nonsusceptible 35B/ST558. During 1995-2001, the penicillin-nonsusceptible 35B/ ST558 lineage, which had resistant MICs of $0.25-2.0 \mu \mathrm{g} /$ $\mathrm{mL}$, accounted for $69 \%$ of serotype 35B ABCs isolates (3). During 1999-2007 in the United States, the proportion of penicillin-nonsusceptible IPD isolates within serotype $35 \mathrm{~B}$ increased to $84 \% ; 35 \mathrm{~B} / \mathrm{ST} 558$ accounted for this increase (4). Consistent with this observation was a 9-fold increase in carriage of 35B/ST558 in young children in the Atlanta, Georgia, area (5).

After introduction of the 13-valent conjugate vaccine (PCV13) in 2010, 35B became the most common serotype in ABCs, associated with MICs $\geq 2 \mu \mathrm{g} / \mathrm{mL}$ for penicillin and amoxicillin in pediatric isolates $(6)$ and in the adult population (B. Beall, unpub. data). Consistent with its status as a major cause of IPD in the post-PCV13 era, the $35 \mathrm{~B} / \mathrm{ST} 558$ lineage is currently commonly found in disease and asymptomatic pneumococcal carriage in many countries $(7-11)$.

We provide a whole-genome sequence (WGS) pipeline-based resolution and description of current 35B lineages within current $\mathrm{ABCs}$ surveillance $(6,12)$, including an invasive $35 \mathrm{~B}$ variant of the historically successful ST156 lineage. Recently, we identified 2 different 35B isolates recovered during 2009 and 2012 that each appeared to have arisen through a unique capsular switch event involving the same 2 parental strains. This observation was made on the basis of the penicillin-binding protein (PBP) gene types flanking the 35B biosynthetic locus (cps35B) in each of the variants $(6)$.

Only the 35B/ST156 variant detected during 2012 has emerged and has been detected within 6 states. ST156 has a remarkable history of conjugate vaccine evasion. Formerly the primary genotype of PCV7 
serotype $9 \mathrm{~V}$ in the United States during the preconjugate vaccine era (13), 9V greatly decreased after PCV7 implementation (1) and was partially replaced by resistant 19A/ST156 (14). To verify that these isolates originated from a single recombinational serotype switch event involving 35B/ST558 and 9V/ST156 parental strains, we analyzed regions flanking the cps $35 B$ locus during 2015-2016, 35B/ST156 progeny and the original strain detected during 2012.

\section{Methods}

\section{Isolates}

The surveillance population of $\mathrm{ABCs}$ is $\approx 32$ million persons in 10 states (http://www.cdc.gov/pneumococcal/ surveillance.html). Serotype 35B IPD isolates described include 132 recovered during 2015 and 67 recovered during ABCs in 2016 (Table 1, https://wwwnc.cdc.gov/EID/ article/23/6/17-0071-T1.htm). The listing of 35B isolates from 2016 is incomplete because we typically receive all $\mathrm{ABCs}$ isolates recovered during a given year by the following summer. Relevant ST156 lineage isolates of other serotypes recovered during this and previous periods are shown in Table 2. Total numbers of ABCs 35B isolates recovered during 1999-2015 and categorized by patient age, penicillin MIC, and IPD incidence are shown in Table 3.

\section{WGS and WGS-Based Predictions}

Library construction and sequencing was performed as described (12). WGS accessions for all 199 serotype 35B isolates from 2015-2016, two previous 35B switch strains from previous years, and relevant strains of other serotypes of ST156 from previous years are provided (online Technical Appendix Table, https://wwwnc.cdc.gov/EID/ article/23/6/17-0071-Techapp1.pdf). WGS pipeline data and quality metrics for all isolates are also provided (online Technical Appendix Table). Capsular serotypes, antimicrobial genotypes/phenotypes, MLST, sequence type (ST), and pili (presence or absence) for year 2015-2016 isolates were deduced through our bioinformatics pipeline $(6,12,15)$.

\section{Phylogeny}

Paired-end fastq files were trimmed with Cutadapt version 1.8.1 (17), and draft genome assemblies were

Table 2. Nonserotype 35B isolates of ST156 lineage included in study of penicillin-nonsusceptible 35B pneumococcal isolates causing IPD, United States, 1998-2015*

\begin{tabular}{|c|c|c|c|c|c|c|c|c|c|c|c|c|c|c|}
\hline \multirow{2}{*}{$\begin{array}{l}\text { Serotype/ } \\
\text { MLST type } \\
\text { (no.) } \dagger\end{array}$} & \multirow[b]{2}{*}{ No. } & \multirow[b]{2}{*}{ PBP type } & \multirow[b]{2}{*}{$\begin{array}{c}\text { Non-PBP resistance } \\
\text { determinants } \S\end{array}$} & \multicolumn{10}{|c|}{ Antimicrobial resistance phenotype, MIC, $\mu \mathrm{g} / \mathrm{mL} \mathbb{I}$} & \multirow[b]{2}{*}{$\begin{array}{l}\text { State (year } \\
\text { isolated) }\end{array}$} \\
\hline & & & & Pen & Amo & Tax & $\mathrm{Cft}$ & Cfx & Mer & Ery & $\begin{array}{c}\mathrm{Cli}+ \\
\text { Tet }\end{array}$ & Cot & $\mathrm{Fq}$ & \\
\hline \multirow[t]{5}{*}{ 9V/156 (25) } & 12 & $15: 12: 18$ & $\begin{array}{l}\text { folAl100L, } \\
\text { folPins } 178\end{array}$ & 4 & 2 & 1 & 2 & $>2$ & 0.5 & $\mathrm{~S}$ & $\mathrm{~S}$ & $\mathrm{R}$ & $\mathrm{S}$ & $\begin{array}{l}\text { CA, GA, MD, } \\
\text { MN, NY, TN } \\
(1998-1999)\end{array}$ \\
\hline & 9 & $15: 12: 18$ & $\begin{array}{l}\text { mef, folAl100, } \\
\text { folPins178 }\end{array}$ & 4 & 2 & 1 & 2 & $>2$ & 0.5 & $\mathrm{R}$ & S & $\mathrm{R}$ & S & $\begin{array}{c}\text { CA, CT, MD, } \\
\text { MN, OR, TN } \\
(1998,1999, \\
2015)\end{array}$ \\
\hline & 2 & $15: 12: 18$ & $\begin{array}{l}\text { ermB, tetM, } \\
\text { folAl100L, } \\
\text { folAins } 178\end{array}$ & 4 & 2 & 1 & 2 & $>2$ & 0.5 & $\mathrm{R}$ & $\mathrm{R}$ & $\mathrm{R}$ & S & CA (2015) \\
\hline & 1 & $15: 12: 18$ & $\begin{array}{c}\text { mef, tetM, folAl100L, } \\
\text { folPins } 178\end{array}$ & 4 & 2 & 1 & 2 & $>2$ & 0.5 & $\mathrm{R}$ & S & $\mathrm{R}$ & S & CT (2015) \\
\hline & 1 & $15: 12: 228$ & $\begin{array}{l}\text { Mef, folAl100L, } \\
\text { folPins } 178\end{array}$ & 4 & 2 & 1 & 2 & $>2$ & 0.5 & $\mathrm{R}$ & $S$ & $\mathrm{R}$ & $S$ & MD (2016) \\
\hline \multirow[t]{2}{*}{ 19A/156 (4) } & 3 & 29:12:26 & $\begin{array}{l}\text { mef, folAl100L, } \\
\text { folAins189 }\end{array}$ & 4 & 2 & 8 & 4 & $>2$ & 0.5 & $\mathrm{R}$ & $\mathrm{S}$ & $\mathrm{R}$ & $\mathrm{S}$ & CA,GA (2009) \\
\hline & 1 & $8: 12: 36$ & $\begin{array}{l}\text { mef, folAl100L, } \\
\text { folAins } 189\end{array}$ & 1 & 2 & 0.5 & 0.5 & 2 & 0.25 & $\mathrm{R}$ & S & $\mathrm{R}$ & S & GA (2015) \\
\hline $13 / 156(1)$ & 1 & $15: 12: 173$ & $\begin{array}{l}\text { folAl100L, } \\
\text { folPins178 }\end{array}$ & 1 & 1 & 0.25 & 0.25 & 1 & 0.5 & $\mathrm{~S}$ & $\mathrm{~S}$ & $\mathrm{R}$ & $\mathrm{S}$ & TN (2015) \\
\hline $31 / 156$ (1) & 1 & $15: 12: 18$ & $\begin{array}{l}\text { mef, folAl100L, } \\
\text { folPins178 }\end{array}$ & 4 & 2 & 1 & 2 & $>2$ & 0.5 & $\mathrm{R}$ & $\mathrm{S}$ & $\mathrm{R}$ & $\mathrm{S}$ & MN (2015) \\
\hline
\end{tabular}

*All isolates were positive for pilus PI-type 1 and negative for pilus PI-type 2. IPD, invasive pneumococcal disease; MLST, multilocus sequence type; PBP, penicillin-binding protein; $R$, resistant; $S$, susceptible; ST, sequence type.

†Types that probably arose through serotype switching are indicated in bold.

†See Li et al. (15) and MIC correlates for PBP types (http://www.cdc.gov/streplab/mic-tables.html).

§For a description of WGS-based bioinformatic pipeline for deduction of all features shown, see Metcalf et al. (6,12). For a description of folP insertions (folPins178, folP189), see Figure 1 in Metcalf et al. (12).

TPredicted MICs for $\beta$-lactam antimicrobial drugs were based on transpeptidase domain sequences of PBPs $1 \mathrm{a}, 2 \mathrm{~b}$, and $2 \mathrm{x}$

(http://www.cdc.gov/streplab/mic-tables.html). For penicillin (meningitis only), nonsusceptible is considered $\geq 0.12 \mu \mathrm{g} / \mathrm{mL}$ (16). Currently applied clinical cutoffs are also provided for the other $5 \beta$-lactams shown (16). Where shown, R and S correspond to breakpoint MIC values (16). Amo, amoxicillin; Cft, ceftriaxone; Cfx, cefuroxime; Cli, clindamycin; Cot, cotrimoxazole; Ery, erythromycin; Fq, fluoroquinolones levofloxacin and ciprofloxacin; Mer,

meropenem; Pen, penicillin; Tax, cefotaxime. 
Table 3. Annual incidence and proportions of penicillin-nonsusceptible 35B pneumococcal isolates causing IPD, United States, 1999-2015*

\begin{tabular}{|c|c|c|c|c|c|c|c|c|c|}
\hline \multirow[b]{2}{*}{ Year } & \multirow{2}{*}{$\begin{array}{l}\text { Surveillance } \\
\text { population }\end{array}$} & \multirow[b]{2}{*}{$\% \mathrm{CIS}$} & \multicolumn{2}{|c|}{$\begin{array}{c}\text { No. } 35 \mathrm{~B} \text { isolates from } \\
\text { patients by age, y }\end{array}$} & \multirow{2}{*}{$\begin{array}{c}\text { Relative incidence } \\
\text { of } 35 \mathrm{~B} \text { IPD }\end{array}$} & \multicolumn{3}{|c|}{ Pen MIC, $\mu \mathrm{g} / \mathrm{mL}$} & \multirow{2}{*}{$\begin{array}{c}\text { penNS 35B } \\
\text { isolates/penS 35B } \\
\text { isolates }\end{array}$} \\
\hline & & & $<5$ & $\geq 5$ & & $\geq 2$ & $0.12-1$ & $\leq 0.06$ & \\
\hline 1998 & $17,383,935$ & 86.1 & 3 & 18 & 1.40 & 9 & 5 & 7 & 2.0 \\
\hline 1999 & $18,550,681$ & 87.0 & 2 & 18 & 1.24 & 8 & 3 & 9 & 1.2 \\
\hline 2000 & $19,821,607$ & 86.3 & 4 & 21 & 1.46 & 7 & 9 & 9 & 1.8 \\
\hline 2001 & $22,479,308$ & 88.1 & 2 & 40 & 2.12 & 14 & 18 & 10 & 3.2 \\
\hline 2002 & $25,051,246$ & 87.6 & 2 & 34 & 1.64 & 20 & 8 & 8 & 3.5 \\
\hline 2003 & $25,264,246$ & 91.4 & 11 & 49 & 2.56 & 22 & 26 & 11 & 4.4 \\
\hline 2004 & $27,419,898$ & 87.9 & 15 & 69 & 3.49 & 44 & 25 & 15 & 4.6 \\
\hline 2005 & $27,816,784$ & 89.5 & 11 & 57 & 2.73 & 35 & 18 & 15 & 3.5 \\
\hline 2006 & $28,204,455$ & 86.7 & 1 & 65 & 2.70 & 37 & 15 & 14 & 3.7 \\
\hline 2007 & $28,579,312$ & 87.5 & 5 & 83 & 3.52 & 51 & 23 & 14 & 5.3 \\
\hline 2008 & $28,856,774$ & 86.7 & 12 & 80 & 3.68 & 62 & 16 & 14 & 5.6 \\
\hline 2009 & $29,206,528$ & 89.8 & 8 & 70 & 2.97 & 45 & 16 & 17 & 3.6 \\
\hline 2010 & $29,757,552$ & 90.2 & 4 & 67 & 2.65 & 52 & 14 & 5 & 13.2 \\
\hline 2011 & $30,075,050$ & 90.3 & 11 & 77 & 3.28 & 71 & 7 & 10 & 7.8 \\
\hline 2012 & $30,356,544$ & 90.6 & 13 & 101 & 4.14 & 94 & 14 & 6 & 18.0 \\
\hline 2013 & $30,604,240$ & 88.7 & 15 & 114 & 4.75 & 94 & 25 & 10 & 11.9 \\
\hline 2014 & $31,328,211$ & 88.2 & 16 & 116 & 4.78 & 104 & 22 & 6 & 21.0 \\
\hline 2015 & $31,977,800$ & 92.0 & 10 & 121 & 4.45 & 101 & 24 & 6 & 20.8 \\
\hline
\end{tabular}

${ }^{\star} \mathrm{CIS}$, case isolates serotyped; IPD, invasive pneumococcal disease.

†Estimated cases $/$ million $=$ total $35 \mathrm{Bs} \times 100 / \%$ CIS surveillance population $/ 1,000,000$

constructed by using VelvetOptimiser version 2.2.5 with an optimal kmer value calculated by using VelvetK (18). Core genome single-nucleotide polymorphism (SNP) identification and alignment were performed by using kSNP3.0 (19). A maximum-likelihood phylogenetic tree was generated from the core SNP alignment by using RaxML version 7.3.0 (20). RaxML was run with an ASC GTRGAMMA DNA substitution model and used the Lewis method for ascertainment bias correction. Node support was assessed by using 500 bootstrap replicates.

\section{Conventional MIC Testing and Serotyping}

Serotype 35B isolates recovered during 2015 were subjected to conventional broth dilution testing for determination of antimicrobial MICs. A selection of these isolates were also subjected to conventional serotyping by using CDC typing antisera as described ( 6 ).

\section{Statistical Analyses}

A $\chi^{2}$ test was performed to evaluate differences among groups. This test was performed by using OpenEpi Version 3.01 (http://www.openepi.com/Menu/OE_Menu.htm).

\section{Results}

\section{Increase in Penicillin-Nonsusceptible 35B during the Conjugate Vaccine Era}

During 1998-2001, penicillin-nonsusceptible 35B accounted for $67.6 \%$ (108) of serotype 35B ABCs isolates (Table 3). During 2002-2015, the proportion of penicillinnonsusceptible IPD isolates with serotype 35B increased to $87.7 \%(1,237 ; \mathrm{p}<0.001)$.

\section{Population Snapshot of Ongoing ABCs for 35B IPD, 2015-2016}

Among 2,710 IPD isolates obtained during 2015 and subjected to WGS, 132 (4.9\%) were serotype 35B. Of 1,528 IPD isolates recovered from partial year 2016 IPD surveillance, 67 (4.4\%) were serotype 35B. Most (168/199) of these isolates belonged to penicillin-nonsusceptible clonal complex (CC) 558 (168 isolates) and CC156 (21 isolates) (Figure 1; Table 1). Serotype 35B CC558 and CC156 isolates of all serotypes discussed were uniformly positive for the $\operatorname{rrg} A$ gene (Tables 1,2), which encodes a pilus subunit that functions in epithelial adhesion (22). Ten isolates of long-standing penicillin-susceptible 35B/ST452 (3) were also recovered. Single $35 \mathrm{~B}$ isolates were identified of ST1092, a lineage of conjugate vaccine serotypes $6 \mathrm{~A}$ and 6B (http://pubmlst.org/spneumoniae/) and of ST11818 (highly related to $15 \mathrm{~A} / \mathrm{ST} 63$ ), an antimicrobial-resistant nonvaccine serotype lineage that has increased in the postconjugate vaccine era (4).

\section{CC558 (35B/CC558)}

Of 168 35B/CC558 isolates obtained, 147 were ST558, 20 were single-locus variants (SLVs) of ST558 corresponding to $13 \mathrm{STs}$, and 1 was a double-locus variant (Figure 1; Table 1). Within CC558, only ST558 had SLVs, which is consistent with initial successful establishment of 35B/ ST558 in its ecologic niche and subsequent rare shedding of closely related SLVs (21).

The increased incidence of 35B IPD during the postPCV7 period (2.1-3.7 cases/million population during 20012009 vs. 1.2-1.3 cases/million population during 1998$1999)$ and the post-PCV13 period (3.3-4.8 cases/million 


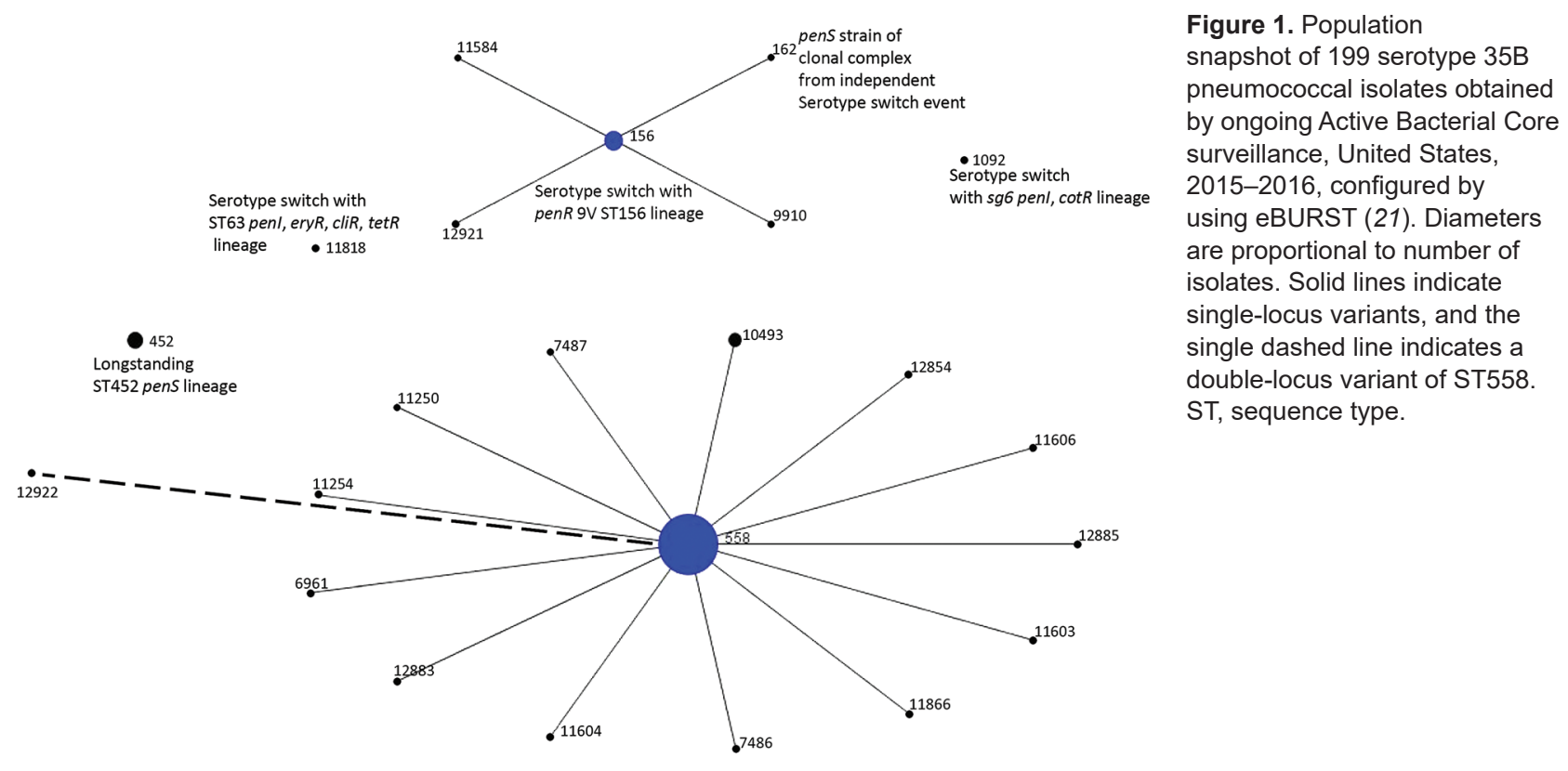

population during 2011-2015), combined with the consistent trend of markedly increased proportions of penicillinnonsusceptible 35B IPD isolates throughout the conjugate vaccine era (Table 2), is consistent with reported increased 35B/ST558 in IPD and carriage (4-11). ABCs surveillance sites increased after 2000, but 35B IPD incidence calculations did not vary whether including the expanded surveillance sites or by using only continuously participating $\mathrm{ABCs}$ sites during 1998-2015.

\section{CC156 (35B/CC156)}

We analyzed PBP types $(6,12,15)$ of 35B/ST558 $(4: 7: 7)$ and 35B/ST156 (4:12:7) isolates. These PBP amino acid sequence types are used for predicting $\beta$-lactam MICs and correspond to PBP transpeptidase domains from PBP1a, PBP2b and PB$\mathrm{P} 2 \mathrm{x}$, respectively. PBP genes $p b p 1 a$ and $p b p 2 x$ flank opposite ends of the capsular biosynthetic locus and are sometimes cotransferred during serotype switching events $(6,23-25)$. The 35B/ST558 lineage has been nearly exclusively associated with PBP type 4:7:7 among isolates obtained since 1998, and the serotype $9 \mathrm{~V} / \mathrm{ST} 156$ lineage is similarly highly associated with PBP type 15:12:18 $(6,15)$. However, 9V/ST156 is rare among IPD isolates in the post-PCV7 period.

In addition to the PBP2b-12 marker, mef gene, and FolA-I100L substitution, candidate cps35B recipient 9V/ ST156 strains contain the 2-codon insertion designated folPins 178 (Table 2; Figures 2, 3). Such 1-2 codon folP insertions, which together with FolA-I100L confer cotrimoxazole resistance, are categorized by specific location of the insertion and specific sequence flanking the insertions (12). These genomic features are also found within the 35B/ ST156 lineage isolates described (Table 1; Figures 2, 3), which are consistent with a 9V/ST156 (mef, FolA-I100L,
folPins 178) strain serving as the recipient strain for a 35B/ ST558 cps35B donor strain (Figure 3). Another potential recipient strain present before and after PCV13 introduction was 19A/ST156 $(6,14)$. However, this lineage is associated with the folPins 189 insertion (Table 2; Figure 2).

Both flanking $p b p$ loci from 35B/ST558 were cotransferred with the cps $35 B$ locus to replace the cps $9 \mathrm{~V}$, pbp $1 a-15$ and $p b p 2 x$-18 determinants in the putative 9V/ST156 recipient, which resulted in PBP type 4:12:7 (Figure 3). This serotype switch progeny strain was obtained from a 4-yearold child during 2012 and is a potential progenitor of the current invasive 35B/CC156 lineage (isolate 2012221165) (Figures 2, 3). Antimicrobial resistance markers PBP2b-12, mef, FolA-I100L, and folPins 178, combined with the close phylogenetic relatedness of the 9V/ST156 isolates (Figure 2 ), suggest that a member of this lineage served as the recipient parental strain for the 35B/ST156 clade isolated in ongoing ABCs during 2015-2016 (Figure 3).

Analysis of the regions flanking the $\operatorname{cps} 35 B$ locus for all 35B/ST156 lineage isolates obtained during 2015-2016 showed identical recombinational sites at bases 6,453 (left coordinate of progeny reference) (Figure 3) and 10,836 (right coordinate), which is clearly indicative of a single event within a 35B/ST156 ancestral strain of the 19 progeny shown (Figure 3). Thus, a double-crossover event replaced the recipient strain cps $9 \mathrm{~V}$ locus and flanking PBP markers (2x-18 and 1a-15) with the cps $35 B$ locus and its flanking PBP markers (2x-7 and 1a-4). On the basis of available strain data, the original progeny strain is predicted to have been an ST156 strain with the PBP type 4:12:7; a total of 14 of the 19 35B/ST156 lineage strains still shared these characteristics (Table 1). Five isolates are SLVs or differ in PBP2b type. 
Figure 2. Phylogenetic analysis of potential recipient and serotype switch pneumococcal progeny strains within the ST56 lineage based upon a total alignment of 10,409 core single-nucleotide polymorphisms, United States, 2015-2016. All 20 serotype 35B progeny shown were recovered through Active Bacterial Core surveillance, and all but 2 indicated strains were obtained during 2015-2016. Isolate features are depicted for the 2 major nodes, with exceptions indicated by asterisks within the tree. Bootstrap values are indicated at key nodes. The serotype $9 \mathrm{~V}$ isolate that was used for the reference recipient sequences described in Figure 3 is indicated as the third isolate from bottom. All isolates above the $9 \mathrm{~V}$ recipient reference within this major cluster, except where indicated, are also 9V/ST156. Three additional serotype switch ST156 strain types detected by Active Bacterial Core surveillance during 2015-2016 are indicated by asterisks (single isolates of serotypes 31 and 13 and 4 serotype 19A isolates). Scale bar indicates nucleotide substitutions per site.

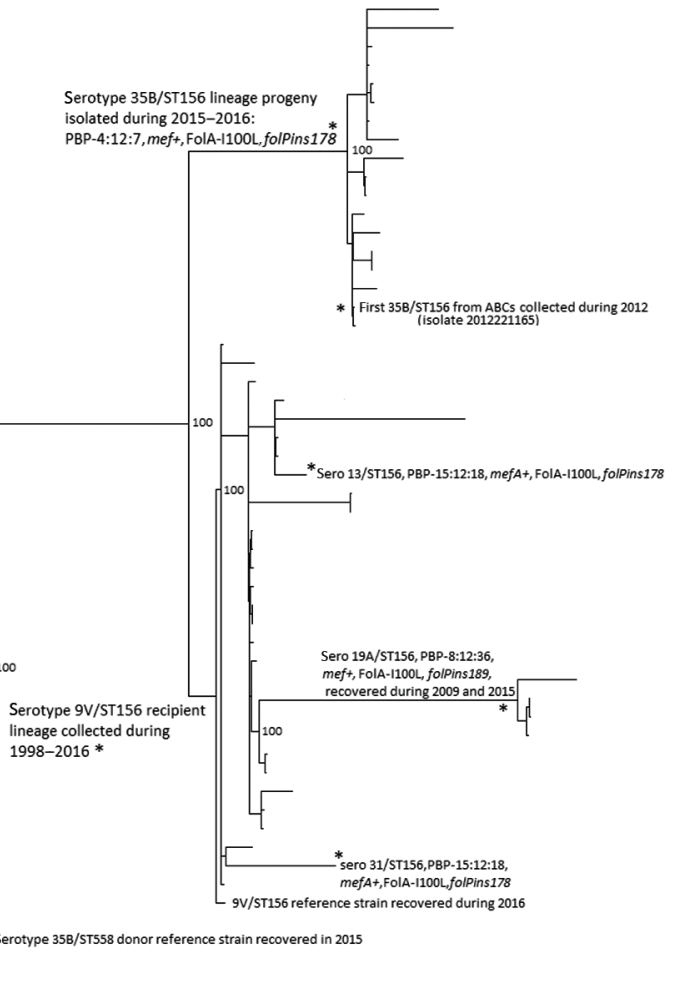

We detected the small (491 bp) segment (bases 11349 11839 of progeny) (Figure 3 ) of clearly recipient lineage origin within the left side of the major recombinational fragment. During a single-double crossover event that facilitates a serotype switch, additional independent doublecrossover events appear to occur concurrently (27). However, these events probably do not occur simultaneously. It appears that the actual serotype switch event involved a shorter donor fragment bordering upon the right side of this small recipient lineage segment (base 11839), followed by a second double-crossover event that bordered upon the left side of the recipient lineage fragment (base 11349).

A single penicillin-susceptible 35B/ST162 (SLV of ST156) has the completely sensitive PBP type 0:0:0 $(6,12)$ (Table 1). This strain arose through an independent serotype switch event that involved a penicillin-susceptible recipient strain.

\section{Postserotype Switch Event Diversification of 35B/ST156 Progeny}

Five of the 19 35B/ST156 lineage progeny showed indications of genetic diversification that occurred after the capsular switch event. Four of these isolates have 1 of 3 SLV MLSTs of ST156 (ST9910, ST11584, and ST12921) (Table 1). Although 18 of the 19 strains were PBP type 4:12:7, the SLV ST12921 variant had PBP type 4:11:7. For this particular strain, it is probable that recombination with a highly penicillin-resistant ST320 strain, prevalent during the post-PCV7 era and having PBP type 13:11:16 (6), simultaneously replaced the $p b p 2 b$ locus and flanking $d d l$ sequence to change the PBP type to 4:11:7 and the MLST type to ST12921 through transfer of $d d l$ with the resistanceconferring selectable $p b p 2 b$ allele (28). We also observed an increased MIC for amoxicillin for this PBP type 4:11:7 strain compared with MICs for PBP type 4:12:7 strains (Table 1).

Two isolates (20152877 and 20161763) underwent a postswitch intra-cps35B gene deletion event within the $w c i G$ gene (Figure 3), which is predicted to encode an acetyltransferase (26). Although these 2 isolates were serotyped as $35 \mathrm{~B}$ by using CDC typing antisera, they differed in reactivity with serologic factor 35 a compared with the other 17 isolates of this lineage (Table 4). Typing antisera factors $29 \mathrm{~b}$ and $35 \mathrm{c}$ are the CDC Quellung reagents definitive for serotype $35 \mathrm{~B}$. The original protocol $(29,30)$ that CDC first followed for serogroup 35 resolution also used factor $35 \mathrm{a}$ along with factors $29 \mathrm{~b}$ and $35 \mathrm{c}$ for identification of serotype 35B. We found that the $2 w c i G$ deletion strains did not react with factor $35 \mathrm{a}$, but the other 17 serotype 35B strains reacted strongly with factor $35 \mathrm{a}$. These preliminary data is suggestive of a new serotype within serogroup 35 because this specific factor reactivity pattern has not been observed for serogroup $35(31)$. 

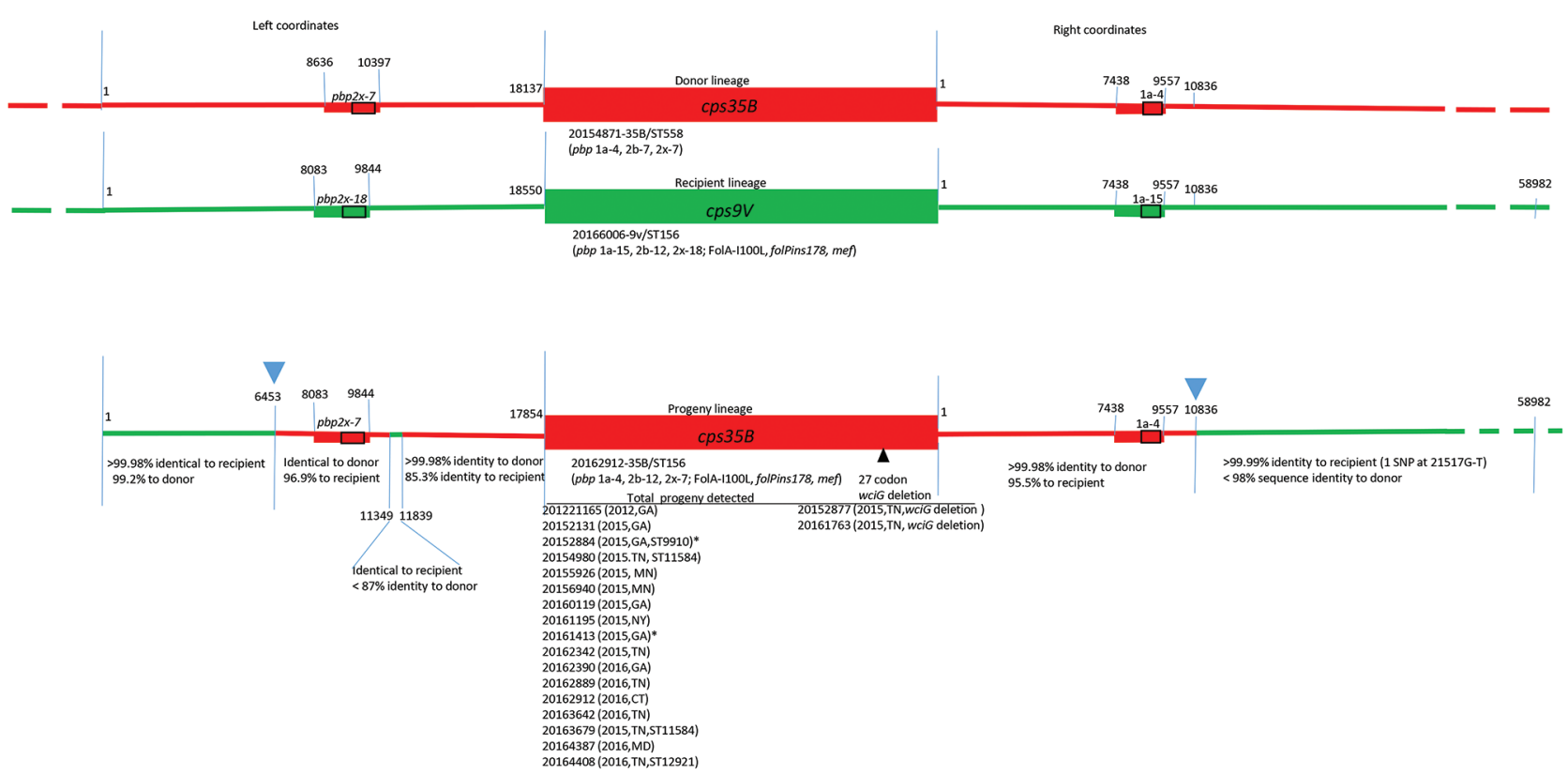

Figure 3. Diagrammatic representation of $c p s$ loci and adjacent regions from donor, recipient and progeny strains depicting serotype switch event for pneumococcal isolates, United States, 2015-2016. Red and green lines in progeny indicate regions of sequence identity or near identity ( $<2$ single-nucleotide polymorphisms/10,000 bp) to the above corresponding donor and recipient sequences, respectively. Rectangles indicate relative locations of PBP gene types for $p b p 2 x$ and $p b p 1 a$. Below each cps locus, a representative reference strain is indicated along with relevant features determined through a bioinformatics pipeline (MLST, PBP type, resistance markers). Junctions between donor and recipient sequences involved in the 2 single recombinational crossovers in the gene replacement event are indicated with blue arrowheads above the progeny diagram, although a single short internal region with sequence identity to the recipient nested within the donor fragment (left coordinates 11349-11839) is also present. Below each green or red segment of the progeny, the level of sequence identity to donor and recipient is provided. The list of each progeny strain, date of isolation, and state is provided. Where MLST is not ST156, its single locus variants (ST9910, ST11584, and ST12921) are included. Two exceptions indicating flanking post-switch recombination within left coordinates 1-6453 are indicated in isolates 20152884 and 20161413 (asterisks): isolate 20152884 had only $99.3 \%-99.5 \%$ identity to recipient and donor over bases $1-3715$, and isolate 20161413 had only $99.5 \%-99.7 \%$ identity to recipient and donor over bases 1-2143. Two strains on the right indicate a post-switch deletion event within the wciG putative acetyltransferase gene, which putatively contributes to the acetylation pattern of the serotype 35B polysaccharide (26). MLST, multilocus sequence type; PBP, penicillin-binding protein; ST, sequence type.

Further indication of chromosome-wide postswitch diversification of this single clade was shown in the leftflanking region of the $c p s 35 B$ locus. Two progeny strains showed diversification within the first $2-3.7 \mathrm{~kb}$ when compared with the other 17 progeny. The $6-\mathrm{kb}$ region immediately to the left of base $1 \mathrm{in}$ all of the progeny strains had $\leq 99.2 \%$ sequence identity with the most similar potential ST156 parental recipient strains that we analyzed. However, beyond this segment, progeny had sequence identity with the parental strain for $\geq 8 \mathrm{~kb}$.

\section{B/ST156 Variant Lineages Arising through Separate Serotype Switch Events}

The 9V/ST156 clade also appears likely to have served as the recipient for an independent serotype switch from the same 2 parental strains (Figure 3) which resulted in 35B/ST10174, an SLV of ST156 (Figure 2) obtained from an infant during 2009 (isolate 2009219987). This isolate differs in the flanking $p b p 2 x$ marker and distal $p b p 2 b$ marker (PBP type 4:7:18). Features of this variant have been described (6), and we have not obtained additional 35B isolates with these distinguishing features. A third serotype switch event involving a CC156 recipient strain is intuitive from the pipeline data, which indicate that the $35 \mathrm{~B}$ SLV of ST162 is featured by the $\beta$-lactam-susceptible PBP type 0:0:0 (Table 1). ST162 has long been associated with penicillin-susceptible 9V strains (13) and more recently with PI-1 positive and penicillin-susceptible 23B, $15 \mathrm{~B}$, and $15 \mathrm{C}$ strains (6).

\section{Nonserotype 35B Variants of ST156}

Single ST156 isolates of serotypes 31 and 13 were obtained during 2015 (Figure 2) and showed high relatedness to different $9 \mathrm{~V}$ subdivisions. Again, the likely recipient background for the presumed capsular switch does not appear likely to involve the 19A/ST156 lineage (Figure 2), which 
Table 4. Serologic comparison of 35B/ST156 lineage strains with CDC Quellung reagents for resolution of serogroup 35B invasive serotype pneumococci including an expanding serotype switch lineage, United States, 2015-2016*

\begin{tabular}{lccccc}
\hline & \multicolumn{5}{c}{ Quellung factor } \\
\cline { 2 - 6 } Strain & $35 \mathrm{~b}$ & $29 \mathrm{~b}$ & $35 \mathrm{c}$ & $42 \mathrm{a}$ & $35 \mathrm{a}$ \\
\hline cps35B† & - & + & + & - & + \\
$c p s 35 B$ (wciG deletion) $\ddagger$ & - & + & + & - & - \\
\hline${ }^{*} \mathrm{CDC}$, Centers for Disease Control and Prevention; ST, sequence type; \\
,- negative; +, positive. \\
†Refers to 17 progeny resulting from recombination indicated in left \\
column under progeny lineage diagram in Figure 3. \\
†Refers to 2 indicated wciG deletion isolates in right column under \\
progeny lineage diagram in Figure 3.
\end{tabular}

was well-represented during the 2000s after PCV7 implementation $(6,14,32)$.

\section{CCs of Remaining 35B Isolates Obtained during 2015-2016}

Ten of the 12 isolates other than penicillin-nonsusceptible CC558 and CC156 (together composed of 187 isolates) were of the long-established penicillin-susceptible 35B/ ST452 lineage (3), which decreased in proportion during the 2000s (4) (Figure 1; Table 3). The 2 remaining 35B isolates obtained during 2015-2016 also appear to have originated through serotype switching events involving a 35B/ST558 cps35B donor, as implicated by the presence of the PBP1a-4 or PBP2x-7 determinants flanking the cps loci of these 2 progeny strains $(35 \mathrm{~B} / \mathrm{ST} 11818$ and 35B/ ST1092) (Table 1). The 35B/ST11818 variant is an SLV of ST63 and has the same resistance features and accessory resistance genes (ermB and tet $M$ ) as the currently common $15 \mathrm{~A} / \mathrm{ST} 63$ clone $(4,6,32)$. The 35B/ST1092 isolate is likely to have originated through serotype switching with a serogroup 6 recipient strain (6). The 14 remaining ST1092 isolates obtained during surveillance in 2015-2016 were serotype $6 \mathrm{C}$. The 6 previously collected ST1092 IPD isolates (1999-2013) represented in our WGS collection are from serotype $6 \mathrm{~A}, 6 \mathrm{~B}$, and $6 \mathrm{C}$ strains (6; B. Beall, unpub. data).

\section{Discussion}

An increase of penicillin-nonsusceptible serotype 35B IPD and carriage caused by $35 \mathrm{~B} / \mathrm{ST} 558$ has been apparent in the United States since the introduction of PCV7 in 2000, and it has shown a major increase after PCV13 implementation (4-8). This finding is of concern because even strains that are rarely detected in IPD sometimes rapidly emerge. For example, the 19A/ST320 strain was not detected during extensive characterization of pre-PCV7 $\mathrm{ABCs}$ isolates (13), yet it became the predominant invasive pneumococcal strain during 2005-2009 (14,32). We have performed comprehensive strain characterization (MLST and WGS) of pediatric (from children $\leq 5$ years of age) ABCs isolates obtained during 1999, 2001, 2002, 2008, 2009, and 2011$2013(6,13)$ and WGS-based characterization of a large sampling of isolates from all age groups during 1998-2013 $(4,6,13$; B. Beall, unpub. data).

Before 2015, we detected only 1 isolate of the 35B/ ST156 lineage that was recovered during 2012 (6). Thus, we feel justified in describing it as newly emergent isolate. A smaller study was recently published (during peer review of this article) that described 78 invasive and 48 noninvasive serotype 35B isolates obtained during 1994 2014 from 8 hospitals in 8 states (33). Our data, which included a population-based sampling of 199 35B isolates obtained during 2015-2016, clearly shows the current national predominance of 35B/ST558 and does not support the observation that $35 \mathrm{~B} / \mathrm{ST} 156$ is the major contributor to post-PCV13 antimicrobial-resistant 35B. Both studies noted the initial appearance and emergence of $35 \mathrm{~B} / \mathrm{ST} 156$ in the post-PCV13 period.

This recent identification of the antimicrobial-resistant 35B/ST156 lineage and its subsequent detection within 6 ABCs sites is a cause for concern. The ST156 lineage has shown a remarkable propensity to persist through undergoing serotype-switch events $(12,23,25,32)$. The penicillinresistant 9V/ST156 lineage was the predominant serotype $9 \mathrm{~V}$ cause of IPD in the United States during the pre-PCV7 era $(13,32)$. Soon after introduction of PCV7, serotype 9V IPD became rare $(1,2)$, and 19A became the predominant representative of the ST156 lineage within ABCs $(14,32)$. After introduction of PCV13, 35B has become the predominant serotype of the ST156 lineage within the United States (B. Beall, unpub. data).

A distinct antimicrobial-susceptible serotype 35B SLV of ST156 (35B/ST162) is included among 35B ABCs of 35B during 2015-2016 by the $\beta$-lactam-susceptible PBP type 0:0:0. Thus, our data indicates that $\geq 3$ independent serotype switches involving the nonvaccine type cps $35 B$ locus and the broad ST156 clonal complex serving as recipient strains have previously occurred. In this study, we demonstrated that all penicillin-nonsusceptible 35B/ST156 lineage isolates obtained during current ABCs (2015-2016) arose through a single ancestral recombination event. This event was facilitated through detailed analyses of crossover points and comparisons of corresponding regions of all progeny isolates with likely parental 35B/ST558 and 9V/ ST156 strains. The genetic plasticity of the ST156 lineage is also highlighted in this study by detection of postserotype switch changes affecting $\beta$-lactam resistance (PBP1a type) and capsular serotype (wciZ deletion), which is potentially reflective of recent antimicrobial drug pressure and immunologic selection pressure.

An additional 35B variant within a vaccine serotype lineage is shown with ST1092 that is typically associated with serogroup 6 strains (Figure 1). Because these putative 35B switch variants were not detected during extensive strain surveillance before and shortly after conjugate 
vaccine implementation $(3,4,13)$, it is plausible that these serotype switches occurred after implementation of conjugate vaccine. The observation of a $35 \mathrm{~B}$ variant within the antimicrobial 15A/ST63 lineage brings the number of serotype switch events generating $35 \mathrm{~B}$ strains described in this study to $5 ;(35 \mathrm{~B} / \mathrm{ST} 11818,35 \mathrm{~B} / \mathrm{ST} 156,35 \mathrm{~B} / \mathrm{ST} 162$ from 2015-2016, and 35B/ST10174 from 2009). Except for the 35B/ST162 variant, these serotype switch events were predicted on the basis of progeny PBP type to involve the 35B/ ST558 strain as the cps35B donor (online Technical Appendix Table).

Although conjugate vaccines have a history of providing effective and durable protection against $\operatorname{IPD}(1,2)$, the continued emergence and expansion of serotype 35B into different clonal complexes supports continued development of wider spectrum pneumococcal vaccines. Serotype 19A IPD, although relatively uncommon in the pre-PCV7 era, rapidly became the predominant invasive serotype in the post-PCV7 period $(14,32,34)$. Serotype 35B strains have several of the same features that were found among serotype 19A strains before implementation of PCV7 in 2000. These features that could predispose for serotype 35B to continue its increasing trend as a cause of IPD include its lack of inclusion within conjugate vaccine, high carriage rates within children, antimicrobial resistance, clonal expansion, and serotype switching. An experimental 15-valent conjugate vaccine in development includes serotypes $22 \mathrm{~F}$ and $33 \mathrm{~F}(35)$, which have increased as causes of IPD in the postconjugate vaccine era. Serotypes $15 \mathrm{~A}, 15 \mathrm{~B}$, and $23 \mathrm{~A}$ are expressed by moderately antimicrobial-resistant clones and are not uncommon causes of IPD $(4,32)$. Although less resistant to $\beta$-lactam antimicrobial drugs than 35B/ST558 and 35B/ST156, these strains also present a challenge to address through more encompassing pneumococcal vaccines.

\section{Acknowledgments}

We thank A. Reingold, S. Brooks, H. Randel, L. Miller, B. White, D. Aragon, M. Barnes, J. Sadlowski, S. Petit, M. Cartter, C. Marquez, M. Wilson, M. Farley, S. Thomas, A. Tunali, W. Baughman, L. Harrison, J. Benton, T. Carter, R. Hollick, K. Holmes, A. Riner, Ruth Lynfield, Anita Glennen, C. Holtzman, R. Danila, K. MacInnes, K. Scherzinger, K. Angeles, J. Bareta, L. Butler, S. Khanlian, R. Mansmann, M. Nichols, N. Bennett, S. Zansky, S. Currenti, S. McGuire, A. Thomas, M. Schmidt, J. Thompson, T. Poissant, W. Schaffner, B. Barnes, K. Leib, K. Dyer, L. McKnight, R. Gierke, O. Almendares, J. Hudson, L. McGlone, Tamara Pilishvili, G. Langley, and Cynthia Whitney for their contributions to establishment and maintenance of the ABCs system; Rebecca Gladstone, Stephen Bentley, and Paulina Hawkins for providing genome sequences through the Global Pneumococcal Sequencing Project (http://www.pneumogen.net) from isolates obtained during 1998-1999, 2009, and 2012; the Minnesota Department of Public Health laboratory for performing susceptibility testing of all isolates from Minnesota; and Robert E. Gertz, Jr for providing insights into CDC pneumococcal typing serum factors.

This study was supported by the Centers for Disease Control and Prevention and used data from the Streptococcus pneumoniae MLST website (http://pubmlst.org/_spneumoniae) at the University of Oxford (Oxford, UK).

Development of the Streptococcus pneumoniae MLST website was supported by the Wellcome Trust.

Dr. Chochua is a researcher in the Streptococcus Laboratory, National Center for Immunization and Respiratory Diseases, Centers for Disease Control and Prevention, Atlanta, GA. Her research interests are next-generation sequencing, characterization of clinical streptococcal isolates, antimicrobial resistance, genetic adaptations, and outbreak responses.

\section{References}

1. Pilishvili T, Lexau C, Farley MM, Hadler J, Harrison LH, Bennett NM, et al.; Active Bacterial Core Surveillance/Emerging Infections Program Network. Sustained reductions in invasive pneumococcal disease in the era of conjugate vaccine. J Infect Dis. 2010;201:32-41. http://dx.doi.org/10.1086/648593

2. Moore MR, Link-Gelles R, Schaffner W, Lynfield R, Lexau C, Bennett NM, et al. Effect of use of 13-valent pneumococcal conjugate vaccine in children on invasive pneumococcal disease in children and adults in the USA: analysis of multisite, populationbased surveillance. Lancet Infect Dis. 2015;15:301-9. http://dx.doi.org/10.1016/S1473-3099(14)71081-3

3. Beall B, McEllistrem MC, Gertz RE Jr, Boxrud DJ, Besser JM, Harrison LH, et al.; Active Bacterial Core Surveillance/Emerging Infections Program Network. Emergence of a novel penicillinnonsusceptible, invasive serotype 35B clone of Streptococcus pneumoniae within the United States. J Infect Dis. 2002;186:11822. http://dx.doi.org/10.1086/341072

4. Gertz RE Jr, Li Z, Pimenta FC, Jackson D, Juni BA, Lynfield R, et al. Increased penicillin-nonsusceptibility of nonvaccine serotype (other than 19A and 6A) invasive pneumococci in post 7 valent conjugate vaccine era. J Infect Dis. 2010;201:770-5. http://dx.doi.org/10.1086/650496

5. Sharma D, Baughman W, Holst A, Thomas S, Jackson D, da Gloria Carvalho M, et al. Pneumococcal carriage and invasive disease in children before introduction of the 13-valent conjugate vaccine: comparison with the era before 7-valent conjugate vaccine. Pediatr Infect Dis J. 2013;32:e45-53. http://dx.doi.org/10.1097/INF.0b013e3182788fdd

6. Metcalf BJ, Gertz RE Jr, Gladstone RA, Walker H, Sherwood LK, Jackson D, et al.; Active Bacterial Core surveillance team. Strain features and distributions in pneumococci from children with invasive disease before and after 13-valent conjugate vaccine implementation in the USA. Clin Microbiol Infect. 2016;22:60. e9-29. http://dx.doi.org/10.1016/j.cmi.2015.08.027

7. Desai AP, Sharma D, Crispell EK, Baughman W, Thomas S, Tunali A, et al. Decline in pneumococcal nasopharyngeal carriage of vaccine serotypes after the introduction of the 13 -valent pneumococcal conjugate vaccine in children in Atlanta, Georgia. Pediatr Infect Dis J. 2015;34:1168-74. http://dx.doi.org/10.1097/ INF.0000000000000849 
8. Kaur R, Casey JR, Pichichero ME. Emerging Streptococcus pneumoniae strains colonizing the nasopharynx in children after 13 -valent pneumococcal conjugate vaccination in comparison to the 7-valent era, 2006-2015. Pediatr Infect Dis J. 2016;35:901-6. http://dx.doi.org/10.1097/INF.0000000000001206

9. Kawaguchiya M, Urushibara N, Kobayashi N. Multidrug resistance in non-PCV13 serotypes of Streptococcus pneumoniae in northern Japan, 2014. Microb Drug Resist. 2017;23:206-14. http://dx.doi.org/ 10.1089/mdr.2016.0054

10. McElligott M, Vickers I, Meehan M, Cafferkey M, Cunney R, Humphreys H. Noninvasive pneumococcal clones associated with antimicrobial nonsusceptibility isolated from children in the era of conjugate vaccines. Antimicrob Agents Chemother. 2015;59:57617. http://dx.doi.org/10.1128/AAC.00990-15

11. Golden AR, Adam HJ, Gilmour MW, Baxter MR, Martin I, Nichol KA, et al. Assessment of multidrug resistance, clonality and virulence in non-PCV-13 Streptococcus pneumoniae serotypes in Canada, 2011-13. J Antimicrob Chemother. 2015;70:1960-4.

12. Metcalf BJ, Chochua S, Gertz RE Jr, Li Z, Walker H, Tran T, et al.; Active Bacterial Core surveillance team. Using whole genome sequencing to identify resistance determinants and predict antimicrobial resistance phenotypes for year 2015 invasive pneumococcal disease isolates recovered in the United States. Clin Microbiol Infect. 2016;22:1002.e1-8. http://dx.doi.org/ 10.1016/j.cmi.2016.08.001

13. Beall B, McEllistrem MC, Gertz RE Jr, Wedel S, Boxrud DJ, Gonzalez AL, et al.; Active Bacterial Core Surveillance Team. Pre- and postvaccination clonal compositions of invasive pneumococcal serotypes for isolates collected in the United States in 1999, 2001, and 2002. J Clin Microbiol. 2006;44:999-1017. http://dx.doi.org/10.1128/JCM.44.3.999-1017.2006

14. Beall BW, Gertz RE, Hulkower RL, Whitney CG, Moore MR, Brueggemann AB. Shifting genetic structure of invasive serotype 19A pneumococci in the United States. J Infect Dis. 2011;203: 1360-8. http://dx.doi.org/10.1093/infdis/jir052

15. Li Y, Metcalf BJ, Chochua S, Li Z, Gertz RE Jr, Walker H, et al. Penicillin-binding protein transpeptidase signatures for tracking and predicting $\beta$-lactam resistance levels in Streptococcus pneumoniae. MBio. 2016;7:e0756-16. http://dx.doi.org/10.1128/ mBio.00756-16

16. Clinical and Laboratory Standards Institute (CLSI). Performance standards for antimicrobial susceptibility testing. Twenty-third informational supplement (M100-S22.CLSI). Wayne (PA): The Institute; 2013

17. Martin M. Cutadapt removes adapter sequences from highthroughput sequencing reads. EMBnet. 2011;17:10-2. http://dx.doi.org/10.14806/ej.17.1.200

18. Zerbino DR, Birney E. Velvet: algorithms for de novo short read assembly using de Bruijn graphs. Genome Res. 2008;18:821-9. http://dx.doi.org/10.1101/gr.074492.107

19. Gardner SN, Slezak T, Hall BG. kSNP3.0: SNP detection and phylogenetic analysis of genomes without genome alignment or reference genome. Bioinformatics. 2015;31:2877-8. http://dx.doi.org/10.1093/bioinformatics/btv271

20. Stamatakis A. RAxML version 8: a tool for phylogenetic analysis and post-analysis of large phylogenies. Bioinformatics. 2014;30:1312-3. http://dx.doi.org/10.1093/bioinformatics/btu033

21. Feil EJ, Li BC, Aanensen DM, Hanage WP, Spratt BG. eBURST: inferring patterns of evolutionary descent among clusters of related bacterial genotypes from multilocus sequence typing data. J Bacteriol. 2004;186:1518-30. http://dx.doi.org/10.1128/ JB.186.5.1518-1530.2004
22. Barocchi MA, Ries J, Zogaj X, Hemsley C, Albiger B, Kanth A, et al. A pneumococcal pilus influences virulence and host inflammatory responses. Proc Natl Acad Sci U S A. 2006;103:2857-62. http://dx.doi.org/10.1073/pnas.0511017103

23. Coffey TJ, Daniels M, Enright MC, Spratt BG. Serotype 14 variants of the Spanish penicillin-resistant serotype 9V clone of Streptococcus pneumoniae arose by large recombinational replacements of the cpsA-pbpla region. Microbiology. 1999; 145:2023-31. http://dx.doi.org/10.1099/13500872-145-8-2023

24. Brueggemann AB, Pai R, Crook DW, Beall B. Vaccine escape recombinants emerge after pneumococcal vaccination in the United States. PLoS Pathog. 2007;3:e168. http://dx.doi.org/ 10.1371/journal.ppat.0030168

25. Wyres KL, Lambertsen LM, Croucher NJ, McGee L, von Gottberg A, Liñares J, et al. Pneumococcal capsular switching: a historical perspective. J Infect Dis. 2013;207:439-49. http://dx.doi.org/10.1093/infdis/jis703

26. Mavroidi A, Aanensen DM, Godoy D, Skovsted IC, Kaltoft MS, Reeves PR, et al. Genetic relatedness of the Streptococcus pneumoniae capsular biosynthetic loci. J Bacteriol. 2007; 189:7841-55. http://dx.doi.org/10.1128/JB.00836-07

27. Golubchik T, Brueggemann AB, Street T, Gertz RE Jr, Spencer CC, Ho T, et al. Pneumococcal genome sequencing tracks a vaccine escape variant formed through a multi-fragment recombination event. Nat Genet. 2012;44:352-5. http://dx.doi.org/10.1038/ng.1072

28. Enright MC, Spratt BG. Extensive variation in the $d d l$ gene of penicillin-resistant Streptococcus pneumoniae results from a hitchhiking effect driven by the penicillin-binding protein 2b gene. Mol Biol Evol. 1999;16:1687-95. http://dx.doi.org/ 10.1093/oxfordjournals.molbev.a026082

29. Kauffmann F, Mørch E, Schmith K. On the serology of the pneumococcus-group. J Immunol. 1940;39:397-426.

30. Lund E. On the nomenclature of the pneumococcal types. Int J Syst Bacteriol. 1970;20:321-3. http://dx.doi.org/10.1099/ 00207713-20-3-321

31. Henrichsen J. Six newly recognized types of Streptococcus pneumoniae. J Clin Microbiol. 1995;33:2759-62.

32. Kim L, McGee L, Tomczyk S, Beall B. Biological and epidemiological features of antibiotic-resistant Streptococcus pneumoniae in pre- and post-conjugate vaccine eras: a United States perspective. Clin Microbiol Rev. 2016;29:525-52. http://dx.doi.org/10.1128/CMR.00058-15

33. Olarte L, Kaplan SL, Barson WJ, Romero JR, Lin PL, Tan TQ, et al. Emergence of multidrug-resistant pneumococcal serotype 35B among children in the United States. J Clin Microbiol. 2017;55:724-34. http://dx.doi.org/10.1128/JCM.01778-16

34. Moore MR, Gertz RE Jr, Woodbury RL, Barkocy-Gallagher GA, Schaffner W, Lexau C, et al. Population snapshot of emergent Streptococcus pneumoniae serotype 19A in the United States, 2005. J Infect Dis. 2008;197:1016-27. http://dx.doi.org/10.1086/ 528996

35. Sobanjo-ter Meulen A, Vesikari T, Malacaman EA, Shapiro SA, Dallas MJ, Hoover PA, et al. Safety, tolerability and immunogenicity of 15 -valent pneumococcal conjugate vaccine in toddlers previously vaccinated with 7-valent pneumococcal conjugate vaccine. Pediatr Infect Dis J. 2015;34:186-94. http://dx.doi.org/10.1097/INF.0000000000000516

Address for correspondence: Bernard Beall, Centers for Disease Control and Prevention, 1600 Clifton Rd NE, Mailstop C02, Atlanta, GA

30329-4017, USA: email bbeall@cdc.gov 